\title{
The Ongoing Challenge of Acute Mesenteric Ischemia
}

\author{
Bernd Luther $^{\mathrm{a}} \quad$ Apostolos Mamopoulos $^{\mathrm{b}}$ Christian Lehmann $^{\mathrm{c}}$ Ernst Klar $^{\mathrm{d}}$ \\ a Department of Vascular Surgery, Maria Hilf Alexianer Hospital, Krefeld, Germany; \\ ${ }^{b}$ Department of Vascular Surgery, Helios Hospital, Krefeld, Germany; \\ ${ }^{\mathrm{c}}$ Department of Anesthesia, Dalhousie University, Halifax, NS, Canada; \\ dDepartment of General, Thoracic, Vascular and Transplant Surgery, University Rostock, Rostock, Germany
}

\section{Keywords}

Acute mesenteric ischemia - Emergency procedure .

Endovascular mesenteric reconstruction .

Surgery of mesenteric arteries - Ischemic bowel disease

\section{Summary}

Background: The lethality of acute mesenteric ischemia (AMI) remains quite high with $50-70 \%$. The main reasons for that are the fact that AMI is rarely taken into consideration by the differential diagnosis of acute abdomen, the time-consuming diagnostic process, and the lack of a standardized therapeutic concept. The present interdisciplinary review aims to increase awareness among physicians and to help improve clinical outcomes. Methods: This clinical therapeutic review is based on author expertise as well as a selective literature survey in PubMed based on the term 'mesenteric ischemia', combined with the terms 'arterial', 'clinical presentation', 'diagnosis', 'therapy', 'surgery', and 'interventional radiology'. Based on these search results as well as on the guidelines of the German Society of Vascular Surgery, the American College of Cardiology, and the American Heart Association, we present an interdisciplinary treatment concept. Results: AMI is a vascular emergency that can be successfully treated only within the first hours after the onset of symptoms. Computed tomography angiography is the diagnostic method of choice. Intensive care unit treatment can prevent the occurrence of multiple organ failure. Treatment primarily consists of the revascularization of the mesenteric arteries. Endovascular techniques should be given priority, whereas signs of peritonitis or a central arterial occlusion with high thrombus load primarily require a surgical approach in order to save time and increase patient safety. Additional bowel resections can play a significant role in the treatment of intestinal sepsis. Conclusion:
Prompt and goal-oriented diagnosis and consistent treatment of AMI within 4-6 $\mathrm{h}$ from the onset of symptoms can be decisive for the reduction of AMI-associated lethality. In order for this to happen, a standardized concept of emergency treatment needs to be implemented.

(c) 2018 S. Karger GmbH, Freiburg

\section{Introduction}

Acute mesenteric ischemia (AMI) remains an interdisciplinary challenge in clinical medicine. The symptoms can be non-specific and the diagnosis takes time, resulting in delayed treatment with high lethality. With increasing awareness among physicians, primarily in the fields of gastroenterology as well as abdominal and vascular surgery, however, a reduction of AMI-associated mortality from 90 to $50 \%$ could be achieved in the last 30 years [1] Among patients over 70 years of age with the diagnosis 'acute abdomen', AMI is found to be the cause of symptoms in $10 \%$ [2]. Considering that treatment within the first $6 \mathrm{~h}$ results in a mortality of only $10-20 \%$, it becomes obvious that the potential of improved outcomes is quite high.

The objective of this comprehensive review of all aspects of AMI is to increase the understanding of this clinical entity among physicians. It has to be clear that AMI is a cardiovascular emergency that requires strict decision pathways similar to those established for cerebrovascular or acute myocardial ischemia. For this purpose, we present our own treatment algorithm. Characteristic for AMI is the sudden onset of abdominal pain initially, a subsequent pain-free interval of $6 \mathrm{~h}$, followed by sepsis with multiple organ failure after that, resulting in a challenging situation for the intensive care unit (ICU). This review is based on a literature survey in PubMed of the term 'mesenteric ischemia' as well as on the current guidelines of the German Society of Vascular Surgery (GSVS), the American College of Cardiology (ACC), and the American Heart Association (AHA).

\section{KARGER}

(๑) 2018 S. Karger GmbH, Freiburg

Fax +497614520714 


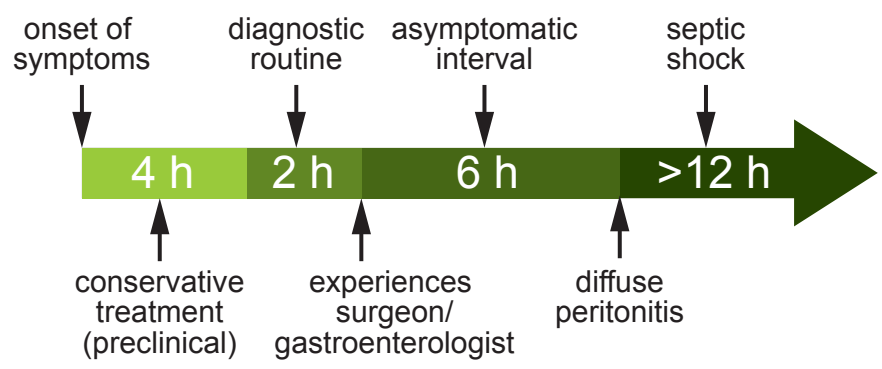

Fig. 1. Clinical course and treatment of acute mesenteric ischemia (according to $[30])$.

\section{Causes and Classification}

AMI is the result of a sudden occlusion of one or more principal mesenteric arteries. It has been shown that the anatomic configuration of the celiac artery (CA), the superior mesenteric artery (SMA), and the inferior mesenteric artery (IMA) forms a collateral network with a high compensatory capacity [3]. Certain limitations exist for the SMA, however, since this artery is only centrally collateralized, resulting in a functionally vulnerable peripheral circulation. This vessel is affected in $85 \%$ of all cases of AMI, and it plays the main role in the diagnosis of AMI. The pathogenesis of the occlusion can be embolic or thrombotic in the presence of a pre-existing pathological arterial wall. Additionally, central occlusion should be distinguished from peripheral occlusion. Often cited risk factors for an AMI are cardiac arrhythmias, mostly atrial fibrillation, older myocardial infarction, general arteriosclerosis, and arterial hypertension. A separate type of a functional, non-occlusive mesenteric ischemia (NOMI) is described, which can result from a reactive vascular spasm within the intestinal circulation. It can occur in low-flow situations, e.g. at the end of cardiac surgery procedures or during dialysis. Septicemia or certain drugs (digitalis) can also induce a NOMI.

\section{Pathophysiology and Clinical Aspects}

An acute complete occlusion of a mesenteric artery initially triggers a vascular spasm in the area of the ischemic bowel. The result is hyperperistalsis with paroxysmal abdominal pain. A vicious circle is simultaneously established on the cellular level, which results in energy loss with the formation of oxygen free radicals and the subsequent disintegration of mucosal cells. After 3-6 h, the intestinal peristalsis ceases and a deceptive mild interval without pain begins, which results from the ischemic damage of the intramural pain receptors. In the final stage the mucosal defect becomes irreversible. Along with the infiltration of the intestinal wall by leukocytes, bacterial translocation takes place, resulting in intestinal gangrene. The clinical manifestation of the end stage consists of diffuse peritonitis, ileus, sepsis, and multiple organ failure, especially failure of the lungs, kidneys, and liver (fig. 1). Because of this clinical course, the need for an early recognition of AMI in the initial stage (usually by the general practitioner) as well as an urgent, consistent clinical diagnosis and prompt revascularization are of the upmost importance.
Table 1. Differential diagnosis of acute mesenteric ischemia

\begin{tabular}{ll}
\hline Abdominal pathologies & $\begin{array}{l}\text { intestinal occlusion or perforation, } \\
\text { inflammation of intra-abdominal organs, } \\
\text { biliary colic, incarcerated hernia }\end{array}$ \\
\hline Vascular pathologies & $\begin{array}{l}\text { aortic aneurysm, aortic dissection, } \\
\text { mesenteric vein thrombosis, portal vein } \\
\text { thrombosis, vasculitis }\end{array}$ \\
\hline Urinary tract pathologies & $\begin{array}{l}\text { renal colic, urinary retention, testicular } \\
\text { torsion }\end{array}$ \\
\hline Traumatic pathologies & blunt force trauma \\
\hline Gynecological pathologies & extrauterine pregnancy, tubal rupture \\
\hline General medicine pathologies & $\begin{array}{l}\text { diabetic pseudoperitonitis, acute } \\
\text { intermittent porphyria, lead poisoning }\end{array}$ \\
\hline Thoracic pathologies & $\begin{array}{l}\text { pleurisy, pericarditis, acute myocardial } \\
\text { infarction }\end{array}$ \\
\hline
\end{tabular}

\section{Diagnosis, Differential Diagnosis and Prognostic Parameters}

The consideration of AMI in the differential diagnosis of 'acute abdominal pain' is decisive for the diagnostic pathway (table 1).

When an AMI is suspected, a biphasic computed tomography (CT) angiography is the diagnostic method of choice and should be performed without delay. The method is currently universally available, is time-saving, and has a high diagnostic value for all intra-abdominal pathologies. Sensitivity is $93 \%$ and specificity is $100 \%$, while the positive and negative prognostic value is 94 and $100 \%$, respectively [4]. A CT scan immediately enables many treatment options of both abdominal und vascular pathologies, with the additional option of an endovascular intervention. If colorectal ischemia is suspected, endoscopic evaluation of the colorectal mucosa should be promptly performed. Other diagnostic means, e.g. abdominal ultrasound, abdominal X-ray, catheter angiography, or magnetic resonance tomography, are usually time-consuming and do not offer a comprehensive presentation of all abdominal organs (ultrasound: class III recommendation with grade $\mathrm{C}$ evidence level according to the ACC/AHA guidelines). These methods should be replaced by CT angiography.

Time-consuming laboratory tests are non-productive as well because of the lack of a marker with adequate sensitivity or specificity that can confirm the diagnosis. This is also the case for serum lactate, with elevated levels being indicative but not evidentiary of an AMI. There are cases of AMI with normal levels of serum lactate, particularly in patients with colorectal or peripheral ischemia [5].

Any delay of the initial diagnosis and intestinal revascularization plays a critical role according to the prognostic parameters (table 2). The lethality of AMI rises exponentially from 10 to $100 \%$ within $24 \mathrm{~h}$ (fig. 2).

Additional predictors arise from the pathogenesis and location of the arterial occlusion. Arterial embolism, for instance, usually 
Table 2. Prognostic parameters of acute mesenteric ischemia

Time lapse till revascularization

Pathogenesis of the occlusion (embolism, thrombosis, NOMI)

Affected intestinal artery (celiac artery, SMA, IMA, multiple vessels)

Location of the occlusion (central, peripheral)

Degree of ischemia (complete, reversible)

Extent of ischemia (extensive, localized)

Affected intestinal segment (small or large intestine, combined)

NOMI = Non-occlusive mesenteric ischemia; SMA = superior mesenteric artery; IMA = inferior mesenteric artery.

occurs in healthy and unprepared vascular areas, promptly leading to a severe defect. On the contrary, an arterial thrombosis usually occurs after a long and chronic atherosclerotic process within the wall of an intestinal artery, resulting initially in a more moderate clinical course with the final stage being just as grave, though. Regarding the localization of the occlusion, the occluded principal vessel is of high importance. While acute occlusions of the CA and the IMA are often adequately compensated by the collateral perfusion of the SMA, the occlusion of the latter almost invariably results in an intestinal infarction. Occlusions of central vessels are generally prognostically less favorable than peripheral occlusions with a better collateral perfusion. The prognosis of NOMI is particularly grim (lethality of 50-70\%), the reason being, on the one hand, the vascular spasm of extensive intestinal segments and, on the other hand, the particularly difficult diagnosis [8]. Finally, the degree and the extent of ischemia as well as the affected intestinal segments should be considered. If no irreversible damage of the intestinal wall is present, a complete regeneration without bowel resection is possible after revascularization. We should also point out that the course of an AMI of the large intestine is more fulminant than that of an AMI of the small intestine. This is the reason why a combined ischemia of the small and large intestine has a higher lethality than an isolated AMI of the small intestine.

Additional prognostic factors include the age and the life quality as well as possible comorbidities of the patient. It is of prognostic value whether the patient is 'young' and relatively 'healthy' or multimorbid with a reduced life expectancy. Treatment options should be oriented towards the preservation or reacquisition of a realistic quality of life and should not represent an end in itself. This narrow line between agonizing preservation of life and realistic treatment optimism is determined by the physician.

\section{Treatment Options}

AMI represents a vascular emergency. Clinical concepts have to be developed and be available in order to reduce the mortality due to the disease, similar to the concepts of the stroke unit for neurologic emergencies or the chest pain unit for patients with myocardial infarction. A comprehensive concept for the emergency management of abdominal patients in Germany is yet to be established.

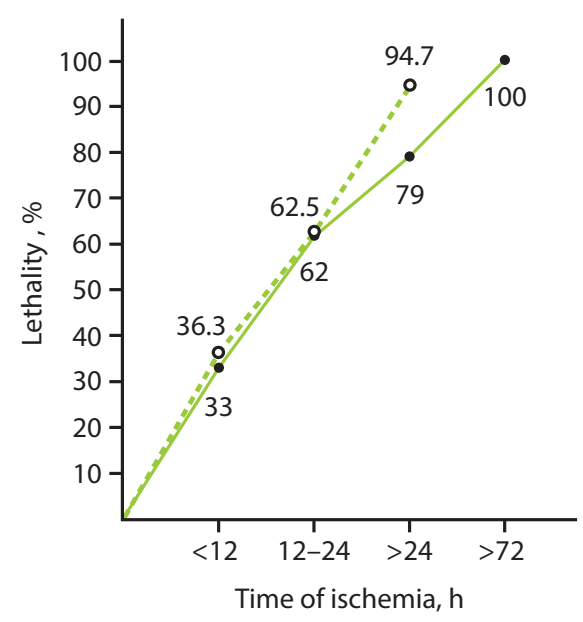

\section{Goals of Basic Intensive Care}

ICU capacity should be available for every patient with suspected AMI from the moment they are admitted to the hospital, provided there is no delay of the diagnostic process or the initiation of revascularization. Continuous measurements of the oxygenation by pulse oximetry, arterial blood pressure, and heart rate present the basic monitoring in those patients [9]. Particular attention should be given to adequate fluid resuscitation with crystalloid solutions as well as blood derivatives to achieve a hemodynamic stabilization of the patient by counteracting the massive volume displacement within the ischemic intestinal segments [1], which takes place in the early hours after the onset of AMI (table 3). In order to achieve that goal, patients should receive a central venous catheter (internal jugular or subclavian vein). Vasopressors should be avoided during this stage, although they could be indicated particularly in high cardiac risk patients in order to avoid a volume overload. Dobutamine, low-dose dopamine, or milrinone improve cardiac function while causing minimal impairment of intestinal blood flow [10]. Supraphysiological parameters for the improvement of cardiovascular function are not an established concept [11]. Volume overload can lead to abdominal compartment syndrome; therefore, the intra-abdominal pressure should be clinically monitored and if a compartment syndrome is suspected, it should be confirmed by measurement of the intravesical pressure. The administration of oxygen should be part of the routine. Fasting should be initiated immediately, both as preparation for a potential surgical procedure and to reduce the postprandial intestinal hyperemia.

At the same time, anticoagulant therapy with heparin should be initiated in order to prevent the progression of the thromboembolic occlusive process within the intestinal arteries. We recommend an intravenous bolus of 20,000 IU followed by a continuous administration of 5,000-10,000 IU of heparin within $24 \mathrm{~h} \mathrm{[12].} \mathrm{The}$ aPTT should be about $60 \mathrm{~s}$. Because of bacterial translocation from the ischemic bowel segments, caused by the disturbed mucosa barrier, all patients should be given antibiotics, e.g. second-generation cephalosporins combined with metronidazole, even when there are 
Table 3. Basic care of acute mesenteric ischemia

\begin{tabular}{ll}
\hline Fluid resuscitation & e.g. Ringer's lactate $1-2 \mathrm{ml} / \mathrm{kg} / \mathrm{h}$ \\
\hline Anticoagulation & $20,000 \mathrm{IE} / \mathrm{h}$ eparin $/ 24 \mathrm{~h}$, PTT-controlled \\
\hline Antibiotics & cephalosporin $3 \times 1.5 \mathrm{~g}$, metronidazole $3 \times 500 \mathrm{mg}$ \\
\hline Analgesics & $\begin{array}{l}\text { peridural catheter or opioids, e.g. up to } 15 \mathrm{mg} / \mathrm{h} \\
\text { piritramide }\end{array}$ \\
\hline
\end{tabular}

no clinical signs of an infection [13]. A sufficient analgesic therapy is also required.

A particular situation arises in the presence of non-occlusive AMI: ICU patients are often affected as they represent a high-risk group because of the administration of high-dose vasopressors, disturbed blood coagulation, as well as fluid or electrolyte deficits. The symptomatic treatment in this case consists of the improvement of volume substitution in order to achieve a significant dose reduction of vasopressors. Additional measures for a local (intestinal) vasodilation and anticoagulation can be applied [14].

\section{Are Endovascular Techniques a Reasonable Treatment Option?}

In every case of AMI and once the diagnosis has been confirmed, one should evaluate the feasibility and rationality of endovascular treatment (ET). Here, the obvious reduction of patient morbidity is advantageous, particularly since geriatric and multimorbid patients are often those affected. The reason for that is the lack of major surgical trauma (laparotomy, intestinal displacement, vascular clamping, hypothermia etc.). Branco et al. [15] determined a reduction in patient morbidity to $23-26 \%$ after ET compared to $36-56 \%$ after open surgery. Other authors came to similar conclusions, although there were differences in the classification of the severity of the disease $[16,17]$. ET did not result in a significant reduction of the rate of pneumonia or sepsis as well as of the occurrence of short bowel syndrome, so that the overall mortality of AMI could not be reduced $[18,19]$. The technical aspects of target vessel recanalization (usually the SMA) via a femoral, brachial, or, in the case of a laparotomy (peritonitis), retrograde mesenteric access is usually successful. Having a guidewire in the target vessel allows thrombectomy with or without selective thrombolysis using rt-PA or urokinase, respectively, and the administration of prostaglandin $\mathrm{E} 1\left(\mathrm{PGE}_{1}\right)$ locally through the catheter in order to reduce thrombus load [20]. A thrombus fragmentation using the guidewire is not recommended due to the risk of peripheral embolization. Therefore, we recommend an open thrombectomy in cases of fulminant embolism of the central SMA (fig. 3).

Alprostadil $\left(\mathrm{PGE}_{1}\right.$, Prostavasin ${ }^{\circledR}$ ) administration results in selective vasodilation and is particularly helpful in cases of NOMI (class I recommendation, evidence level B, ACC/AHA guidelines). Recommended are $20 \mu \mathrm{g}$ alprostadil as bolus and additionally $60-80 \mu$ g over $24 \mathrm{~h}$ as continuous infusion directly into the ostium of the SMA (alternatively: epoprostenol ( $\mathrm{PGI}_{2}$, Iloprost $\left.{ }^{\circledR}\right)$ 5-6 ng/

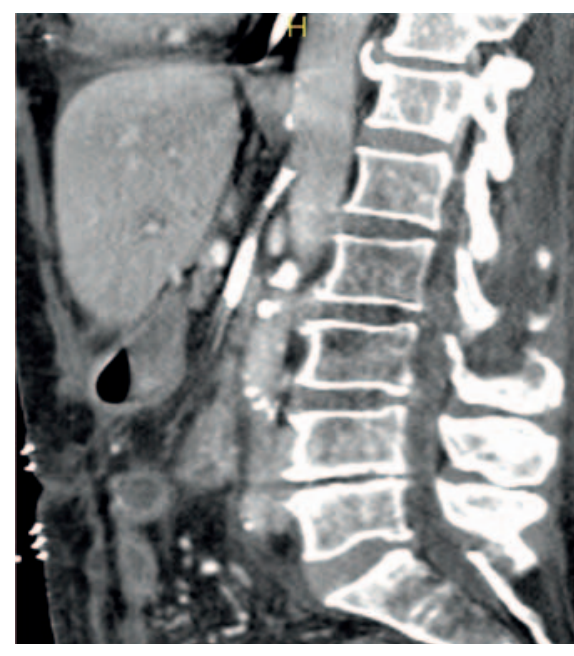

Table 4. Critical lengths of remaining bowel to avoid short bowel syndrome

$100 \mathrm{~cm}$ for a permanent jejunostomy (loss of colon)

$65 \mathrm{~cm}$ for a jejunocolic anastomosis (preservation of colon)

$35 \mathrm{~cm}$ for a jejunoileal anastomosis with preservation of the ileocecal region

$\mathrm{kg} / \mathrm{min}$, or 20,000 IU heparin/24 h). Success of pharmacotherapy should be evaluated by follow-up angiography. Should an underlying stenosis be discovered, an additional stent angioplasty can secure the successful recanalization so that the intestinal reperfusion can be maintained. While balloon-expandable stents can be placed in the proximal SMA, distal stenotic lesions should be treated with self-expanding stents in order to avoid an arterial dissection. The initial technical success rate is $94-100 \%$ [15]. For the proximal SMA, stent diameters of 6-8 $\mathrm{mm}$ are sufficient, while larger-diameter stents are less successful (fig. 4). In order to avoid the consequences of a potential vessel perforation as well as to reduce the rate of late restenosis, stent grafts can be deployed as well [21].

The most common complication after ET, besides access site complications (hematoma, dissection), is peripheral embolization, which is reported in $3.8 \%$ of all cases. Rarer are perforation of the target vessels (19\%), dissections (10\%), as well as acute stent dislocations and acute in-stent thrombosis (10\%) [22]. A significant drawback of ET are the early and late outcomes, which are worse compared to open repair, although they are not relevant for the short-term prognosis of AMI [23]. The implementation of ET should not result in a potential delay of the treatment of intestinal sepsis. That is why the presence of peritonitis should be considered as a contraindication for ET [12].

\section{When Is a Surgical Approach Indicated?}

The surgical treatment of AMI consists of a vascular and abdominal surgical approach so that a combined expertise is always required. The first step is the reperfusion of the mesenteric arteries; the evaluation and potential resection of bowel segments can take place after that. Open vascular repair remains the method of choice 


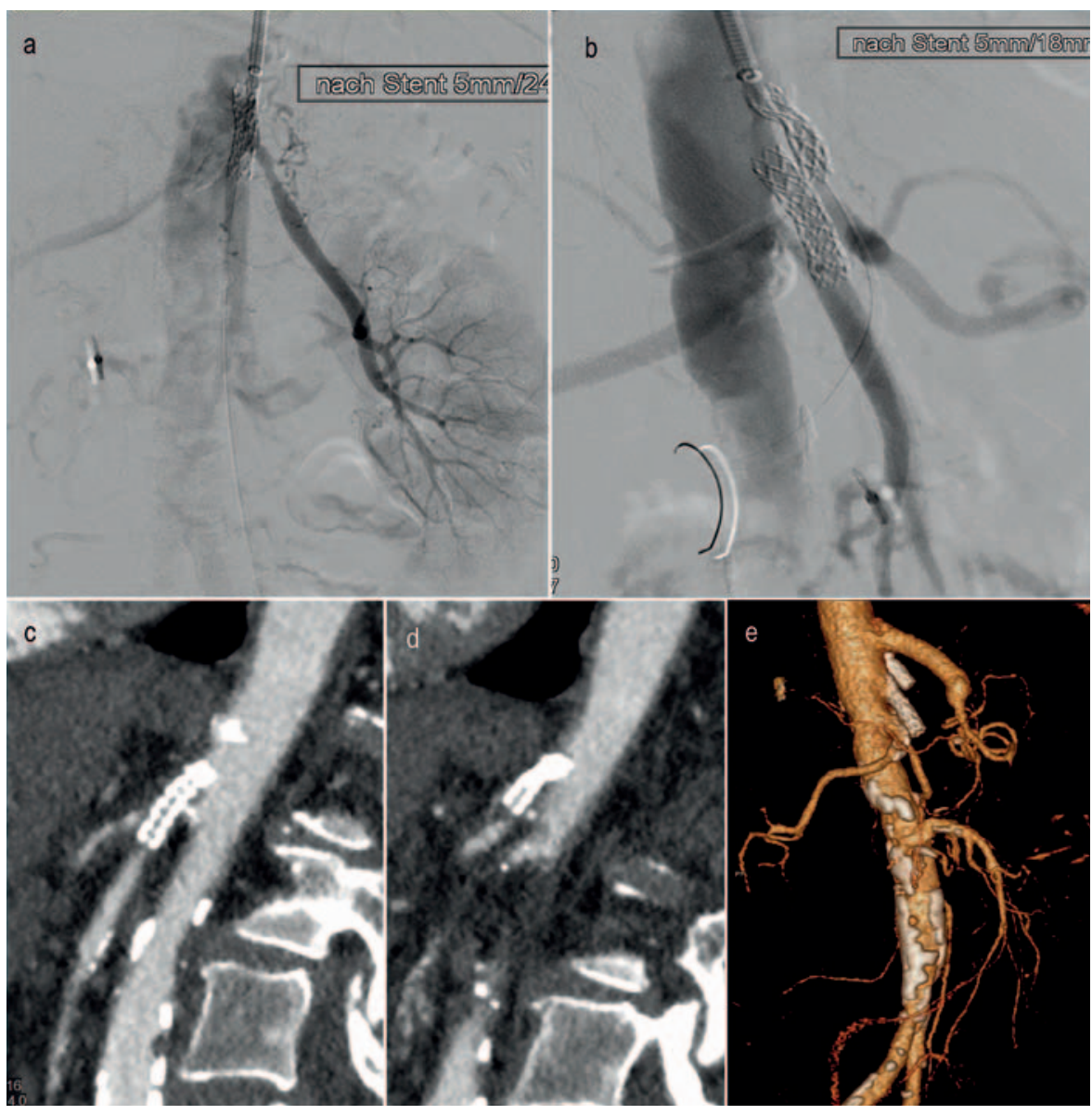

Fig. 4. a, b Implantation of $5 \mathrm{~mm}$ stents in the superior mesenteric artery und celiac artery. c, d Reocclusion of both stents 4 weeks later with acute mesenteric ischemia. e Open reconstruction with an aorto-celiac bypass and aortic reinsertion of the superior mesenteric artery. Outcome: recovery.

in cases of central thromboembolic SMA occlusion, in cases of peritonitis, and as bailout procedure after a failed ET. The technical aspects of transmesenterial embolectomy and of aortic reinsertion of the SMA, as well as the various bypass configurations are adequately described [24]. With sufficient expertise, a primary success rate of $98-100 \%$ can be achieved; thus, time-consuming and uncertain endovascular/hybrid methods with retrograde transmesenterial interventions are not required [25].

In the case of suspected intra-abdominal infection, e.g. bacterial translocation or gangrene, an autologous reconstruction should be preferred in order to avoid an infected prosthesis. Relevant complications include vascular reocclusions (2\%) and bleeding (1\%). The overall morbidity after open vascular repair, however, is higher compared to ET, partially because of the severity of the disease (peritonitis, intestinal resection). The complications of sepsis, such as e.g. pneumonia, kidney failure, abdominal compartment syndrome, result in long-term artificial respiration, catecholamine administration, dialysis, and reoperations.

Once the intestinal revascularization is achieved, the next step is the treatment of the intra-abdominal septic focus. Bowel segments with irreversible damage should be identified and removed. Since the defects of the innermost layers of the mucosa are usually more extensive than the external lesions of the intestinal wall, bowel resections with a primary anastomosis should be undertaken only after careful consideration. In order to avoid the risk of anastomotic insufficiency resulting in an extremely deteriorated prognosis, in uncertain cases one should prefer the exteriorization of the healthy intestinal ends as a stoma [26]. This allows endoscopic follow-up examinations as well as a selective restoration of intestinal continuity after patient recovery. Most favorable for the unstable patient is the resection of ischemic bowel segments with a stapler without further reconstructions, followed by a re-evaluation after 12-24 h (damage control). Should an additional resection of more extensive bowel segments become necessary, one should consider the critical lengths of the remaining bowel (table 4).

If these limits cannot be maintained, the result is a short bowel syndrome, which requires a constant parenteral nutrition or even a bowel transplant. Therefore, when deciding to exceed these resection limits, one should take the age and the comorbidities of the patient into consideration.

\section{Challenges in Postoperative Intensive Care}

Postoperative intensive care of AMI patients is directed towards the control of intestinal ischemia/reperfusion (I/R) and the prevention of a potentially fatal abdominal sepsis. Release of toxic products of local inflammatory processes (e.g. cytokines, oxygen free radicals) into the bloodstream occurs in the initial postoperative 
phase [27]. This can lead to a systemic inflammatory response syndrome with multiple organ failure, even in the absence of a primary intra-abdominal septic focus. In the presence of an additional infection resulting from bacterial translocation from the damaged bowel, a full-blown abdominal sepsis can develop. In addition to basic intensive care with extended hemodynamic monitoring, treatment should include additional measures to stabilize organ functions as well as to control infection, and might require repeated imaging, e.g. X-ray and ultrasound.

In order to reduce the effects of $I / R$, other measures, e.g. antioxidants like acetylcysteine or sodium selenite as well as anti-cytokine therapies, have been suggested [28]. Controlled clinical studies, however, are not available.

Capillary leakage resulting from I/R injury leads to volume sequestration into the ischemic bowel. Therefore, particular attention is required regarding the optimization of volume balance. This becomes more difficult because of the severe I/R-induced systemic hypotension, which often requires catecholamine administration. In this case, and depending on cardiac output and peripheral vascular resistance, a combination of noradrenaline and dobutamine should be considered to minimize the possible negative impact on the intestinal microcirculation [10]. Dialysis, which is often required because of kidney failure, can contribute to hemodynamic stabilization and facilitates the optimization of volume balance.

Because of potential bacterial translocation from the injured gut, sepsis treatment according to the current guidelines should always be initiated [29]. Prolonged ventilation including a tracheostomy is often required.

In the case of persistent clinical instability with increase in infection parameters and bowel segments of uncertain vitality, a second-look procedure should be undertaken $8-12 \mathrm{~h}$ after the primary operation. In this case the indication for a follow-up procedure should be generously given.

\section{Outlook or 'What Lies Ahead?'}

AMI will remain a severe disease with high lethality. The main challenge is the early recognition of the abdominal symptoms by the primary physician, requiring awareness of the disease. Early diagnosis of AMI is hampered by the absence of laboratory parameters specific enough to confirm the presence of AMI. AMI is a vascular emergency and should be diagnosed by a direct approach, thus avoiding time loss. CT angiography is the diagnostic method of choice as the fastest and most reliable modality. A concept for emergency management of AMI, similar to the one presented here, should be implemented in every institution. Parallel to intensive care, invasive treatment methods should be promptly employed. An attempt at endovascular recanalization should be the first step; however, this is only possible in specialized centers. In case of peritonitis or high thrombus load, open surgical procedures must be implemented, because the overall problem of vascular occlusion and gut ischemia can be assessed more reliably and treated faster (fig. 5).

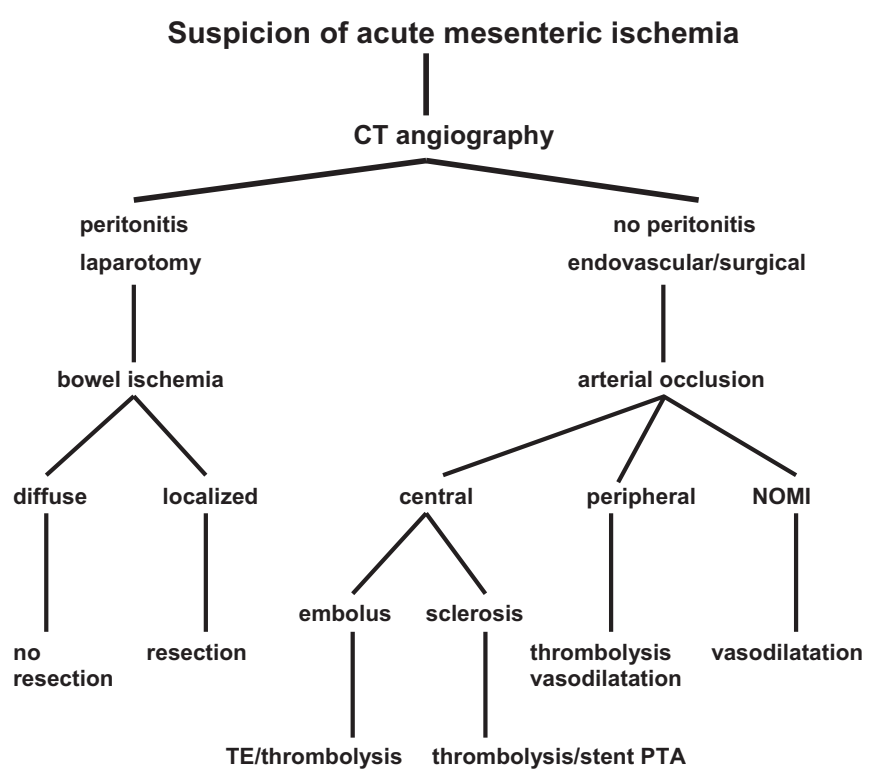

Fig. 5. Diagnosis and treatment algorithm of acute mesenteric ischemia. NOMI $=$ Non-occlusive mesenteric ischemia.

The goal of postoperative intensive care is stabilization of the splanchnic perfusion as well as sepsis prevention/treatment. Surgical goals are the containment of the primary septic source by reexploration and eventual secondary bowel resection. Transition to a palliative concept in all phases of treatment represents an ethical challenge.

\section{Concluding Remarks}

- AMI must be regarded as a vascular emergency!

- Prognosis is time-dependent. Therefore, increased attention should be given to the prompt recognition and efficient diagnosis.

- CT angiography is the method of choice for the identification and further analysis of mesenteric infarction.

- In the early stage, endovascular methods should be implemented due to their minimally invasive character and the lower morbidity.

- In case of peritonitis and high thrombus load, surgical methods should be preferred in order to achieve a simultaneous arterial reconstruction and a resection of irreversibly altered bowel segments as septic source control.

- The postoperative I/R syndrome and potential development of abdominal sepsis require intensive care treatment.

\section{Disclosure Statement}

There is no known conflict of interest involving the authors. This manuscript has not been previously published. 


\section{References}

1 Bala M, Kashuk J, Moore EE, Kluger Y, Biffl W, Gomes CA, Ben-Ishay O, Rubinstein C, Balogh ZJ, Civil I Coccolini F, Leppaniemi A, Peitzman A, Ansaloni L, Sugrue M, Sartelli M, Di Saverio S, Fraga GP, Catena F: Acute mesenteric ischemia: guidelines of the World Society of Emergency Surgery. World J Emerg Surg 2017;12:38.

2 Luther B: Akute viszerale Ischämie. Optionale Möglichkeiten und Realität. Gefässchirurgie 2006;11:167-172.

3 Luther B: Intestinale Durchblutungsstörungen. Mesenterialinfarkt, Angina abdominalis, Therapieoptionen, Prognosen. Darmstadt, Steinkopff, 2001.

4 Aschoff AJ, Stuber G, Becker BW, Hoffmann MH, Schmitz BL, Schelzig H, Jaeckle T: Evaluation of acute mesenteric ischemia: accuracy of biphasic mesenteric multi-detector CT angiography. Abdom Imaging 2009; 34:345-357.

5 Filsoufi F, Rahmanian PB, Castillo JG, Scurlock C, Legnani PE, Adams DH: Predictors and outcome of gastrointestinal complications in patients undergoing cardiac surgery. Ann Surg 2007;246:323-329.

6 Paes E, Vollmar JF, Hutschenreiter S, Schoenberg MH, Schölzel E: Diagnostik und Therapie des akuten Mesenterialinfarktes. Chir Gastroenterol 1990;6:473-480.

7 Walter P, Lindemann W, Koch B, Feifel G: Der akute Mesenterialinfarkt. Klinikarzt 1992;21:457-463.

8 Kammerer S, Köhler M, Schülke C, Lebiedz P, Heinde W, Buerke B: Nichtokklusive mesenteriale Ischämie (NOMI): Moderne diagnostische und therapeutisch interventionelle Strategien aus radiologischer Sicht. Med Klin Intensivmed Notfmed 2015;110:545-550.

9 Wyers MC: Acute mesenteric ischemia: diagnostic ap proach and surgical treatment. Semin Vasc Surg 2010; 23:9-20.

10 Tendler DA, LaMont JT: Acute mesenteric ischemia. Uptodate. 2013. www.uptodate.com/contents/acutemesentericischemia.

11 Helmerhorst HJ, Schultz MJ, van der Voort PH, de Jonge E, van Westerloo DJ: Bench-to-bedside review: the effects of hyperoxia during critical illness. Crit Care 2015;19:284.
Klar E, Rahmanian PB, Bücker A, Hauenstein K, Jauch KW, Luther B: Acute mesenteric ischemia: a vascular emergency. Dtsch Arztebl Int 2012;109:249-256.

13 Silvestri L, van Saene HK, Zandstra DF, Marshall JC, Gregori D, Gullo A: Impact of selective decontamination of the digestive tract on multiple organ dysfunction syndrome: systematic review of randomized controlled trials. Crit Care Med 2010;38:1370-1376.

14 Meilahn JE, Morris JB, Ceppa EP, Bulkley GB: Effect of prolonged selective intramesenteric arterial vasodilator therapy on intestinal viability after acute segmental mesenteric vascular occlusion. Ann Surg 2001;234: 107-115.

15 Branco BC, Montero-Baker MF, Aziz H, Taylor Z, Mills JL: Endovascular therapy for acute mesenteric ischemia: an NSQIP analysis. Am Surg 2015;8:1170-1176.

16 Acosta S: Surgical management of peritonitis secondary to acute superior mesenteric occlusion. World J Gastroenterol 2014;20:9936-9941.

17 Roussel A, Castier Y, Nuzzo A, Pellenc Q, Sibert A, Panis Y, Bouhnik Y, Corcos O: Revascularization of acute mesenteric ischemia after creation of a dedicated multidisciplinary center. J Vasc Surg 2015;62:12511256.

18 Eslami MH, Rybin D, Doros G, McPhee JT, Farber A: Mortality of acute mesenteric ischemia remains unchanged despite significant increase in utilization of endovascular techniques. Vascular. 2016;24:44-52.

19 El Farargy M, Abdel Hadi A, Abou Eisha M, Bashaeb $\mathrm{K}$, Antoniou GA: Systematic review and meta-analysis of endovascular treatment for acute mesenteric ischaemia. Vascular 2017;25:430-438.

20 Schoots IG, Levi MM, Reekers JA, Lameris JS, van Gulik TM: Thrombolytic therapy for acute superior mesenteric artery occlusion. J Vasc Interv Radiol 2005; 16:317-329.

21 Oderich GS, Erdoes LS, Lesar C, Mendes BC, Gloviczki P, Cha S, Duncan AA, Bower TC: Comparison of covered stents versus bare metal stents for treatment of chronic atherosclerotic mesenteric arterial disease. J Vasc Surg 2013;58:1316-1324.
2 Oderich GS, Tallarita T, Gloviczki P, Duncan AA, Kalra M, Misra S, Cha S, Bower TC: Mesenteric artery complications during angioplasty and stent placement for atherosclerotic chronic mesenteric ischemia. J Vasc Surg 2012;55:1063-1071.

23 Luther, B, Meyer F, Mamopoulos A, Zapenko A, Doerbecker R, Wullstein C, Kroeger K, Katoh M: Endovaskuläre Möglichkeiten und Grenzen bei akuten und chronischen mesenterialen Verschlussprozessen. Zentralbl Chir 2015;140:486-492.

24 Luther B: Intestinalarterien; in Luther B (ed): Techniken der offenen Gefäßchirurgie. Standards, Taktiken, Tricks. Berlin, Springer, 2014, pp 199-222.

25 Blauw JT, Meerwaldt R, Brusse-Keizer M, Kolkman JJ, Gerrits D, Geelkerken RH: Retrograde open mesenteric stenting for acute mesenteric ischemia. J Vasc Surg 2014;60:726-734.

26 Unalp HR, Atahan K, Kamer E, Yaşa H, Tarcan E, Onal MA: Prognostic factors for hospital mortality in patients with acute mesenteric ischemia who undergo intestinal resection due to necrosis. Ulus Travma Acil Cerrahi Derg 2010;16:63-70.

27 Grootjans J, Lenaerts K, Buurman WA, Dejong CHC Derikx JPM: Life and death at the mucosal-luminal interface: new perspectives on human intestinal ischemia-reperfusion. World J Gastroenterol 2016;22: 2760-2770.

28 Tassopoulos A, Chalkias A, Papalois A, Iacovidou N, Xanthos T: The effect of antioxidant supplementation on bacterial translocation after intestinal ischemia and reperfusion. Redox Rep 2017;22:1-9.

29 Rhodes A, Evans LE, Alhazzani W, Levy MM, Antonelli M, Ferrer R, Kumar A, Sevransky JE: Surviving sepsis campaign: international guidelines for management of sepsis and septic shock: 2016. Intensive Care Med 2017;43:304-377.

30 Kuehn F, Klar E: Vaskuläre Komplikationen im Darmtrakt. Gastroenterologie Up2date 2014;10:159170. 